Relations industrielles

Industrial Relations

\title{
Le concept du " Welfare State "
}

Essai d'interprétation sociologique

\section{Maurice Tremblay}

Volume 6, numéro 2, mars 1951

URI : https://id.erudit.org/iderudit/1023231ar

DOI : https://doi.org/10.7202/1023231ar

Aller au sommaire du numéro

\section{Éditeur(s)}

Département des relations industrielles de l’Université Laval

\section{ISSN}

0034-379X (imprimé)

1703-8138 (numérique)

Découvrir la revue

\section{Citer cet article}

Tremblay, M. (1951). Le concept du " Welfare State " : Essai d'interprétation sociologique. Relations industrielles / Industrial Relations, 6(2), 42-48.

https://doi.org/10.7202/1023231ar

Tous droits réservés @ Département des relations industrielles de l’Université Laval, 1951
Ce document est protégé par la loi sur le droit d'auteur. L'utilisation des services d'Érudit (y compris la reproduction) est assujettie à sa politique d'utilisation que vous pouvez consulter en ligne.

https://apropos.erudit.org/fr/usagers/politique-dutilisation/ 


\title{
Le concept du "Welfare State"
}

\section{Essai d'interprétation sociologique}

\author{
par Maunce Tremblay, professeur à la Faculté des sciences sociales de Laval
}

Cette étude est consacrée à analyser les problèmes soulevés par l'évolution des démocraties libérales vers le régime du * Welfare State »,* à dégager les facteurs qui sont impliqués dans cette évolution et peuvent contribuer à en rendre compte.

Quelles sont donc les racines sociologiques du *Welfare State»?

Nous croyons qu'elles plongent dans l'Etat libéral tel qu'il s'est constitué au XIXième siècle. Nous soumettons que le «Welfare State se situe dans le prolongement de l'Etat

\footnotetext{
* Pourquoi cette expression anglaise pour désigner l'objet de nos études ? Etymologiquement l'appellation \&Welfare State est pourtant bien vague. Elle pourrait s'appliquer indifféremment à tout régime politique, car il n'en est aucun qui ne réclame pour fin le bien-être des citoyens. L'usage en a cependant précisé le sens et elle est maintenant universellement admise, en langue anglaise, pour désigner le régime particulier d'orientation économique et d'assistance sociale que sont en train d'adopter les démocraties libérales, à mi-chemin entre le régime du «laissez faire » et le régime socialiste.
}

C'est probablement d'ailleurs le vague étymologique de l'expression qui explique son succes, en lui permettant de couvrir, d'une facon indifférencié et sans rien préjuger, tous les aspects de la réalité complexe et controversée qu'elle représente.

Aucune désignation n'a eu la même fortune en langue francaise. Régime de protectorat social, Etat sécuritaire, Etat de service social. Etat paternaliste, Etat-Providence; l'unanimité ne s'est faite sur aucune de ces appellations, probablement parce qu'elles sont toutes trop explicitement péjoratives.

Nous nous en tiendrons donc à l'expression anglaise dont l'usage est fermement établi et qui a le grand avantage, dans une analyse sociologique, de ne rien préjuger a priori. libéral traditionnel, non seulement comme son successeur de fait, mais encore comme son produit, sinon nécessaire du moins normal. Nous soumettons qu'il est né négativement, d'une part, du libéralisme économique, des insatisfactions engendrées par le capitalisme libéral; et positivement d'autre part, du libéralisme politique, du développement même du principe démocratique.

\section{Le libéralisme économique}

Conçu en réaction contre les abus de la réglementation mercantiliste et soutenu par l'exaltation de l'idée de liberté dans tous les domaines, le libéralisme économique se caractérise par la dissociation radicale qu'il opè. re entre l'économique et le politique.

Selon le libéralisme économique, en effet, la prospérité commune n'est pas une fin à laquelle l'Etat doive ordonner rationnellement les divers agents économiques. C'est plutôt une conséquence heureuse qui est appelée à résulter spontanément de la recherche par chacun, en concurrence avec tous les autres, dans les cadres d'un marché libre, de son avantage particulier maximum; et cela, par tous les moyens que peut lui assurer son talent et son industrie, à l'exception toutefois de la violence, de la fraude et de la coalition qui pourrait enrayer le mécanisme de la concurrence.

C'est la concurrence, en effet, qui est censée être le principe animateur et régulateur de l'économie. C'est elle, d'une part, qui doit assurer l'uti-

La Revue des relations industrielles 
lisation maximum des ressources et des énergies productives, en même temps que le perfectionnement continu des produits et des services offerts sur le marché. C'est elle, d'autre part, qui doit assurer l'équilibre du système, en ajustant automatiquement, dans chaque secteur, la production à la consommation, par le jeu de la loi de l'offre et de la demande, qui ne fait qu'exprimer son comportement sur le marché des biens et du travail.

Voici comment Jean Marchal décrit cette dernière fonction attribuée à la concurrence par les tenants du libéralisme économique. «Lorsqu'il y a trop de produits, par suite de la concurrence que se font les vendeurs, les prix s'abaissent. Certains entrepreneurs sont découragés et la production diminue. En même temps, le produit étant rendu accessible à de nouvelles couches d'acheteurs, la demande augmente. Lorsque c'est la demande, au contraire, qui dépasse l'offre, les prix montent, certains acheteurs sont obligés de réduire leur consommation ou de renoncer au produit, tandis que les entrepreneurs, stimulés par les hauts profits, accroissent la production. Des mécanismes identiques se rencontrent en matière de salaires, pour adapter l'offre à la demande de main-d'oeuvre et, en matière d'échanges internationaux pour égaliser les importations et les exportations $\gg .{ }^{1}$

L'économie trouvant ainsi dans les libertés concurrentes son principe à la fois dynamique et régulateur, non seulement l'intervention de l'Etat devient inutile, mais encore elle ne peut contribuer qu'à paralyser et fausser le fonctionnement normal du système. Dans ces conditions, une seule fonction est réservée à l'Etat, celle de veiller au respect de la propriété et des contrats, et d'arbitrer ainsi de l'extérieur, en se contentant de sanctionner les règles du jeu, l'activité

(1) Jean Marchal, Cours d'économie politique, tome I, p. 92, Librairie de Medecis, Paris, 1950. des divers agents économiques. C'est la fameuse politique dite du $\ll$ laissezfaire ou de « l'Etat-Gendarme .

\section{Le capitalisme concurrentiel et ses résultats}

Eh bien, c'est sous le signe de cette conception économico-politique que s'est développé au XIXième siècle le régime capitaliste. Voyons dans quelle mesure les faits ont répondu aux postulats de la doctrine.

On doit d'abord reconnaître que la course libre aux profits par les entrepreneurs capitalistes du XIXième siecle, mettant en oeuvre des techniques toujours plus perfectionnées pour rabaisser leur coût de production et s'assurer contre leurs concurrents une place sans cesse plus avantageuse sur un marché en expansion constante par suite des progrès parallèles des moyens de communication, a provoqué un essor économique sans parallèle dans l'histoire et qu'on a proprement qualifié de révolution: la Révolution industrielle. Jaurès, qui n'est pourtant pas sympatique au régime, admirera \& la puissance d'action révolutionnaire de la bourgeoisie, brisant tous les anciens cadres, dissolvant tous les vieux pouvoirs et toutes les vieilles croyances, bouleversant les habitudes du monde, renouvelant sans cesse sa propre technique, déchaînant la beauté tragique des forces productives illimitées $\gg .^{2}$

Il faut aussi reconnaître que ce déchaînement des forces productives, par la multiplication des moyens de subsistance qu'il a entraînée, a permis un accroissement sans précédent de la population jusque là maintenue, par des moyens de vie primitifs, au niveau du simple renouvellement. En Europe la population passe, au XIXième siècle, de 160 à 400 millions. Aux Etats-Unis, elle saute de 5 à 100 millions et, dans l'ensemble du mon-

(2) J. Jaurès, l'Armée nouvelle, Rieder, p. 306 . 
de, où subsistent pourtant de larges zones que n'a pas touchées le capitalisme, elle s'accroît de 900 à 1600 millions.

Conformément aux implications de la thèse libéraliste, le capitalisme du XIXième siècle a donc vraiment conduit à une production sans cesse accrue et améliorée des biens et des commodités de la vie; par contre il n'a pas rempli les promesses de prospérité commune et d'équilibre économique du libéralisme.

En effet, si le capitalisme en se développant a multiplié les moyens de subsistance et permis, pour autant, un accroissement extraordinaire de la population; par ailleurs, il a maintenu la vaste classe ouvrière qu'il a engendrée au niveau de la simple subsistance, et cela, tant que et dans la mesure où il s'est conformé au principe de la concurrence pure.

La misère ouvrière dans la première moitié du XIXième siècle est bien connue. Salaires misérables, journées de travail démesurément longues, recours inhumain au travail des femmes et enfants, conditions de travail et de vie pitoyables: en un mot tous les éléments d'une misère généralisée.

Cette situation tenait sans doute, par certains de ses aspects, à la jeunesse du régime, à la nécessité, au début d'un processus d'industrialisation, de sacrifier à la production des biens de production celle des biens de consommation; mais elle tenait encore plus fondamentalement au caractère concurrentiel du régime luimême.

* Depuis la suppression des corporations et l'interdiction de toute association ouvrière, écrit Jean Marchal, le prolétaire se présente tout nu et désarmé dans la jungle économique. Il y trouve deux concurrences superposées: la concurrence des patrons entre eux, sur le marché des produits, concurrence qu'ils invoquent et souvent avec raison pour déclarer impossible, sous peine de faillite, toute amélioration des salaires; et, en outre, la concurrence des ouvriers entre eux, sur le marché du travail, où ìs se trouvent isolés, inorganisés, en face de patrons qui, selon l'expression d'Adam Smith, constituent. chacun à soi seul, une coalition naturelle. La classe ouvrière, en fin de compte, fait les frais de toute la concurrence, sur laquelle repose le régime $\star^{3}$

Ainsi donc si la concurrence qui animait le régime a produit de la prospérité, on peut dire qu'elle en a exclu systématiquement la masse ouvrière.

De même la concurrence n'a pas assuré les ajustements automatiques du système économique qu'elle était censée garantir. Des crises de surproductions relatives, génératrices de faillites à la chaîne pour les entrepreneurs et de chômage massif pour les ouvriers, commencent à se produire dès le début du XIXième siècle et elles se sont répétées depuis, à peu près tous les huit ans, avec une régularité étonnante, qui semble bien signifier qu'elles sont un phénomène lié au fonctionnement même du capitalisme industriel.

Si donc, pour résumer la situation pour la majeure partie du XIXième siècle, le jeu des libertés industrielles concurrentes dans l'arène économique a provoqué un développement révolutionnaire de la production, par contre il a créé pour les divers agents économiques un état d'insécurité insupportable contre lequel ils devaient fatalement réagir.

Insécurité des entrepreneurs constamment menacés de dépassement par leurs concurrents et sans défense contre les dépressions économiques.

Insécurité des ouvriers sans cesse dans la crainte d'un renvoi arbitraire ou d'une crise de chômage et incapables de se prémunir avec leurs maigres salaires contre la vieillesse et les hasards de la vie.

Insécurité des agriculteurs qui, dans la mesure où ils produisent maintenant pour le marché, sont eux

(3) Jean Marchal Cours d'économie politique, tome I, p. 97. 
aussi soumis aux risques de la concurrence et aux fluctuations économiques cycliques.

\section{Le processus de consolidation des intérêts économiques}

L'insécurité répugnant de soi à la nature humaine, les uns et les autres devaient essayer de la surmonter. Mais ils ne pouvaient le faire sans travailler et en même temps à restreindre la concurrence à laquelle elle était indissolublement liée. C'est ainsi que de l'insécurité inhérente au capitalisme concurrentiel sont nées, par une espèce de processus dialectique, deux tendances orientées vers sa négation. Une première tendance qui pousse les diverses catégories de producteurs à s'organiser pour dominer le marché chacune dans son secteur. Une seconde tendance qui les incline en même temps à faire appel à la protection de la loi et à l'assistance de l'Etat en vue de consolider leur position sur le marché.

Ces deux tendances, qui s'attaquent aux deux postulats fondamentaux du libéralisme économique: la libre concurrence et son corollaire la non-intervention de l'Etat dans la sphère économique, ont commencé à se manifester dans la seconde moitié du XIXième siècle et n'ont cessé depuis de s'accentuer.

Voyons brièvement comment chacune de ces tendances s'est développée et à quels résultats elle a abouti.

Du côté des entreprises, l'orientation vers le contrôle du marché a pris deux voies, celle de la concentration et celle de l'association.

La concentration a pris elle-même une triple forme.

La concentration horizontale d'abord, en vertu de laquelle, dans les branches les plus importantes de la production, la grande entreprise, sous la forme de monopole ou d'oligopoles, s'est assuré la domination du marché, par suite de l'élimination, de l'absorption ou de l'avortement des entreprises similaires de moindre en- vergure incapables de résister à leur concurrence.

En second lieu, la concentration verticale selon laquelle la grande entreprise, pour se prémunir contre les risques et le coût de la concurrence acheteur-fournisseur, absorbe dans ses structures des entreprises complémentaires situées en haut ou en bas d'elle dans un même processus de fabrication.

Enfin la concentration financière qui par le développement du mécanisme de la société anonyme et du « holding company », permet à un petit nombre d'administrateurs de capitaux de contrôler de vastes secteurs de la vie économique.

Par ailleurs, pour se protéger contre les chutes de prix, les entreprises concurrentes, en dépit de toutes les entraves légales, ont eu de plus en plus recours à l'association, sous formes d'ententes pour fixer les prix, limiter la production et se répartir les débouchés.

Les ouvriers, devaient de leur côté essayer de dominer le marché du travail en tentant d'y éliminer la concurrence qui, au premier stade du capitalisme, les avait tenus dans cette insécurité totale qu'on appelle la misère. Et ç'a été le développement du syndicalisme, favorisé par le groupement des ouvriers et l'éveil de la conscience de classe qui ont suivi le phénomène de concentration des entreprises et d'urbanisation industrielle.

Isolés, les ouvriers étaient condamnés à accepter les salaires misérables et les conditions de travail souvent abjectes que leur concurrence mutuelle et celle des patrons entre eux leur imposaient. Associés, ils sont maintenant en mesure, sous la menace d'un arrêt de travail concerté, de forcer les patrons à majorer leurs salaires et à améliorer leur milieu de travail.

Avec l'extension du syndicalisme, et les appuis légaux et politiques qu'elle a su s'assurer, la classe ouvrière est maintenant de plus en plus capable de faire prévaloir ses reven- 
dications et de substituer ainsi son intervention au mécanisme automatique d'ajustement de la concurrence sur le marché du travail.

La classe agricole a été plus lente à réagir, mais elle aussi tend maintenant de plus en plus, par l'association professionnelle et coopérative à surmonter les hasards de la concurrence dans tout un autre secteur de l'économie.

La même insécurité, qui a poussé les divers agents producteurs à essayer de se prémunir par l'organisation contre la dureté et les à-coups de la concurrence, les a aussi inclinés à faire de plus en plus appel à la protection et à l'assistance de l'Etat dans la lutte économique.

Du côté des entrepreneurs capitalistes, il faut signaler dans la ligne de développement de cette seconde tendance anti-libéraliste la réclamation de tarifs protecteurs contre la concurrence étrangère, les pressions exercées sur l'Etat pour la mise en oeuvre d'une politique colonisatrice ou impérialiste susceptible de fournir à la production nationale un accès aux matières premières et des débouchés assurés, la revendication de subventions gouvernementales au profit d'industries nationales sous-marginales, la demande d'exemption de taxes ou de concessions exclusives pour l'établissement de nouvelles industries et, à l'occasion, le recours à l'Etat pour le renflouement d'entreprises menacées de faillite. D'une façon générale, on peut dire que depuis la fin du 19ième siècle, les entrepreneurs capitalistes ont recours à l'intervention de I'Etat à chaque fois et dans tous les cas où cette intervention, si opposée qu'elle soit aux principes libéralistes, est jugée favorable aux intérêts de leurs entreprises.

La classe ouvrière n'a pas manqué elle aussi de faire appel à l'Etat. Cet appel a été d'autant plus efficace, qu'avec la seconde moitié du XIXième siècle tous les pays capitalistes, dans la ligne du développement du libéralisme politique, passent du régime du suffrage censitaire au régime du suffrage universel. L'Etat libéral avait été jusque là un état bourgeois, contrôlé par les possédants, mais, avec l'extension du droit de vote, il devient un état populaire, obligé de tenir compte des revendications des masses qu'il a incorporées politiquement. Comme le remarque très justement R. M. Maciver ${ }^{4}$ cette évolution, ou plutôt cette révolution politique, n'a pas permis aux capitalistes, propriétaires des moyens de production, de se constituer en classe gouvernante dans le sens où les nobles, propriétaires de la terre, avaient pu le faire.

Quelle que soit l'influence qu'ils exercent sur la politique, celle-ci est désormais appelée à servir aussi les intérêts des autres classes.

Forts de leurs droits politiques les ouvriers s'en sont servis non seulement pour faire reconnaître leur droit à l'association et même à la grève, mais encore pour faire adopter graduellement toute une législation tendant à améliorer leurs conditions de travail et de rémunération.

La classe agricole se prévalant elle aussi de sa puissance politique a suivi la même voie avec le résultat qu'elle est peut-être devenue la classe la plus assistée économiquement par l'Etat.

\section{Emergence du "Welfare State"}

Cependant cette course à l'accroissement et à la stabilisation des revenus des diverses catégories de producteurs, par le double moyen du contrôle du marché et du secours à la protection de l'Etat ne pouvaient pas davantage aboutir à l'équilibre du marché et à la prospérité générale que le régime concurrentiel du début.

En effet, les intérêts des divers groupes d'agents économiques sont

(4) R. M. MacIver, The Modern State, Oxford University Press, Londres, 1941, p. 304 et ss. 
en opposition les uns avec les autres et: leur consolidation ne pouvait qu'accentuer leur antagonisme et conduire à des résultats désastreux pour l'ensemble de l'économie.

Ces résultats se sont concrétisés d'une façon particulièrement tragique lors de la crise de 1929, avec sa paralysie presque totale de la vie économique, son chômage généralisé et l'effrondrement de toutes les catégories de revenus.

Le capitalisme à tendance monopolisatrice ayant fait faillite tout comme le capitalisme concurrentiel, il ne restait qu'un recours pratique, le recours à l'Etat comme agent coordinateur des divers intérêts économiques et premier responsable de la prospérité commune.

Eh bien, c'est la politique particulière adoptée par le gouvernement des démocraties libérales pour faire face à cette nouvelle responsabilité qui caractérise ce qu'on est convenu d'appeler le * Welfare State ».

On peut considérer que le nouveau régime est la résultante de quatre facteurs conjugués.

Il se présente d'abord fondamentalement comme la conséquence des revendications politiques des masses populaires dont les aspirations de plus en plus impératives au bien-être et à la sécurité n'ont pas été satisfaites par le capitalisme libéral.

Il apparaît, en second lieu, comme le résultat de l'évolution organique de l'Etat lui-même qui ne fait que continuer, d'une façon plus systématique et plus compréhensive l'interventionnisme vers lequel les divers groupes de la population l'avaient déjà orienté par leurs demandes sans cesse renouvelées de protection et d'assistance.

Mais il ne suffisait pas que les masses, déçues par le capitalisme libéral, se tournent vers l'Etat comme vers l'agent régulateur de l'économie et le grand promoteur de la prospérité commune. Il ne suffisait pas non plus que celui-ci ait été enclin à assurer ce rôle, il fallait, en outre, que
l'Etat dispose ou croit disposer des moyens proportionnés à cette fonction.

C'est à ce point qu'intervient, comme troisième facteur, dans la constitution du *Welfare State », la Révolution Keynesienne en science économique, pour autant que de la théorie du célèbre économiste on a déduit tout un programme d'action politique destiné à orienter les activités libres des divers agents économiques vers le maintien d'un niveau élevé de l'emploi et du revenu national, de même qu'à assurer la distribution de celuici selon les mérites et les besoins de chacun des citoyens.

L'adoption de cette politique d'inspiration keynesienne, de préférence à une politique socialiste d'étatisation systématique des industries de base et de plannification générale de la vie économique, est par ailleurs fonction d'un quatrième facteur, la survivance dans l'opinion publique des pays démocratiques de l'idéal libéral avec l'attachement qu'il garde au régime de l'entreprise privée et son opposition irréductible à la gérance directe de l'ensemble de l'Economie par l'Etat.

Le «Welfare State » nous apparaît ainsi comme une espèce de compromis institutionnel entre la tradition libérale d'une part et d'autre part la nécessité pratique d'une intervention étatique pour que soient satisfaits les désirs de bien-être et de sécurité de l'ensemble des citoyens.

Le «Welfare State» se concilie avec la tradition libérale dans la mesure où il ne tend pas à régulariser la vie économique par voie d'autorité, mais par voie de compensation, en tâchant d'enrayer, au moyen de son budget, les tendances inflationnaires ou déflationnaires qui menacent sans cesse d'entraîner les divers agents économiques dans une économie de marché libre.

Selon la théorie du *Welfare State » le volume des impôts et des dépenses publiques doit, en effet, varier à contre-courant des fluctuations cy- 
cliques, de façon à stimuler ou à décourager, selon le cas, les placements privés et à maintenir ainsi à un niveau stable l'emploi et le revenu national.

Par contre, pour que cette stabilisation ne s'opère pas à un niveau trop bas, et que ne soit pas compromise, pour autant, la prospérité générale, la théorie comporte qu'on s'applique à assurer aux classes populaires, qui ont le plus de propension à consommer, des revenus qui leur permettent de maintenir une demande suffisante sur le marché des biens et des services.

Le moyen prévu, à cette dernière fin, consiste d'une part dans le dégrèvement des impôts indirects dont l'incidence est générale; et, d'autre part, dans le financement, par l'impôt direct progressif mettant à contribution les épargnes improductives, d'un programme compréhensif de sécurité sociale dont les prestations, en augmentant directement ou indirectement le pouvoir d'achat des masses, doivent assurer une utilisation maximum des forces productives.
L'incidence économique qu'on attend de ces programmes de sécurité sociale dans une politique générale de stabilisation de l'économie de marché à un haut niveau d'emploi et de revenus, ne doit pas cependant nous faire oublier qu'ils sont d'abord inspirés par des préoccupations sociales, par le souci d'assurer aux classes économiquement faibles, ce supplément de bien-être qu'elles réclament et que, même dans une économie de plein emploi, elles ne peuvent s'assurer par leur travail.

Nous tenant aux limites d'une interprétation sociologique, nous espérons avoir démontré que le nouveau régime, avec la conception politicoéconomique dont il s'inspire, est le produit normal de l'Etat libéral du XIXième siècle: qu'il est né d'une part des insatisfactions et de l'insécurité produites par l'application du libéralisme économique; d'autre part, de l'incorporation des masses populaires à l'Etat démocratique dans la ligne de développement du libéralisme politique.

\title{
Les camps de travaux forcés en pays communistes
}

\author{
par Françors BrEghA, de l'Institut tchécoslovaque d l'étranger
}

Dans cette seconde étude, nous essaierons de préciser les raisons réelles de l'établissement des camps de travaux forcés en pays communistes, d'après ce que nous en savons et d'après nos sources d'information.

Les faits cités dans l'étude précédente prouvent sans aucun doute que les CTF ne sont nullement un moyen d'éducation des oisifs, mais bien un moyen de terreur illégal employé par le groupe au pouvoir contre tout le peuple tchécoslovaque. Leur but est, d'une part, la suppression des éléments dangereux ou simplement de ceux dont l'attitude envers le régi- me n'est pas positive, et, d'autre part, l'intimidation de la masse de la population qui pourrait se montrer éventuellement hostile au régime. Ce sont là des raisons générales de l'établissement des camps. Il y a pourtant des raisons directes, immédiates; elles sont, d'une part, d'ordre politique et d'autre part, d'ordre économique.

\section{Raisons politiques}

Les raisons d'ordre politique de l'établissement de la terreur au sein d'un état dictatorial réside dans le 